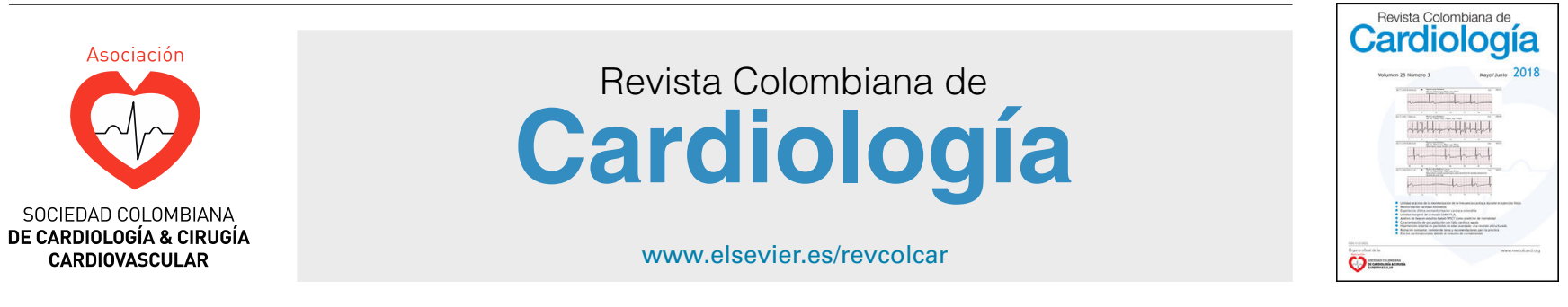

CARDIOLOGÍA DEL ADULTO - ARTÍCULO ORIGINAL

\title{
Validez y confiabilidad de la versión en Español del "Arrhythmia-specific questionnaire in tachycardia and arrhythmia" (ASTA)
}

\author{
Yésica A. Peralta-Linares ${ }^{a, *}$ y Renata V. González-Consuegra ${ }^{\mathrm{b}, c, *}$ \\ a Universidad Nacional de Colombia, Bogotá, Colombia \\ ${ }^{\text {b }}$ Facultad de Enfermería, Universidad Nacional de Colombia, Bogotá, Colombia \\ c ACOFAEN, Asociación Colombiana de Facultades y Escuelas de Enfermería, Bogotá, Colombia
}

Recibido el 15 de junio de 2017; aceptado el 17 de febrero de 2018

Disponible en Internet el 20 de julio de 2018

\author{
PALABRAS CLAVE \\ Arritmias cardiacas; \\ Cuestionarios; \\ Validez; \\ Confiabilidad
}

\begin{abstract}
Resumen
Objetivo: Establecer en español una versión equivalente, válida y confiable del ASTA (Arrhythmia-specific Questionnaire In Tachycardia And Arrhythmia).

Método: Para determinar la validez facial, se aplicó el instrumento a dos grupos: el primero conformado por 7 expertos quienes evaluaron la claridad, compresión y precisión; y el segundo con 453 personas quienes evaluaron la comprensibilidad. Para la validez de contenido, el grupo de expertos evaluó los criterios de pertinencia y relevancia. En la validez de constructo y confiabilidad, 202 pacientes con diferentes arritmias cardiacas diligenciaron el instrumento ASTA.

Resultados: En la evaluación de validez facial con el grupo de expertos, el instrumento reportó un índice de Lawshe mayor a 0,7; para el grupo de personas se obtuvo una comprensibilidad de más del $90 \%$ para 37 ítems. La validez de contenido reportó un índice de Lawshe mayor a 0,8 e índice de kappa de Fleiss mayor a 0,9. La validez de constructo se obtuvo a partir del análisis de factores. Este análisis reveló la presencia de un solo factor para la subescala carga de síntomas, el cual contiene el $43,5 \%$ de la varianza; así mismo, se obtiene un solo factor para la subescala calidad de vida relacionada con la salud, el cual explica el 40,6\% de la varianza. La confiabilidad mediante alfa de Cronbach reportó 0,8.

Conclusión: La versión en español del instrumento ASTA es válida y confiable para evaluar la carga de síntomas y su impacto en la calidad de vida relacionada con la salud de pacientes con arritmias cardiacas.

(c) 2018 Sociedad Colombiana de Cardiología y Cirugía Cardiovascular. Publicado por Elsevier España, S.L.U. Este es un artículo Open Access bajo la licencia CC BY-NC-ND (http:// creativecommons.org/licenses/by-nc-nd/4.0/).
\end{abstract}

\footnotetext{
* Autores para correspondencia.

Correos electrónicos: yaperaltal@unal.edu.co (Y.A. Peralta-Linares), rvgonzalezc@unal.edu.co (R.V. González-Consuegra).
} 


\section{KEYWORDS}

Cardiac arrhythmias;

Questionnaires;

Validity;

Reliability
Validity and reliability of the Spanish version of the Arrhythmia-specific
questionnaire in tachycardia and arrhythmia (ASTA)

\begin{abstract}
Objective: To establish an equivalent, valid and reliable Spanish version of the ASTA (Arrhythmia-specific Questionnaire in Tachycardia and Arrhythmia).

Method: The questionnaire was applied to two groups in order to determine its face validity. The first group consisted of experts that assessed the clarity, conciseness, and precision, and the second with 453 individuals that evaluated its comprehensibility. For the content validity, the expert group evaluated the pertinence and relevance criteria. For the construct and reliability, the ASTA tool was used on 202 patients with different cardiac arrhythmias.

Results: In the evaluation of facial validity with the group of experts, the tool gave a Lawshe value of greater than 0.7 , and for the group of individuals, a comprehensibility of more than $90 \%$ was obtained for 37 items. The content validity gave a Lawshe value greater then 0.8 , and a Fleiss kappa index greater than 0.9 . The construct validity was obtained from the factor analysis. This analysis showed the presence of a single factor for the "symptoms burden" sub-scale, which explained $43.5 \%$ of the variance. Likewise, only one factor was obtained for the "health-related quality of life" sub-scale, which explained $40.6 \%$ of the variance. The reliability, measured by Cronbach alpha, was 0.8 .

Conclusion: The Spanish version of the ASTA questionnaire is valid and reliable for the symptoms burden and the impact on the health-related quality of life of patients with cardiac arrhythmias. (C) 2018 Sociedad Colombiana de Cardiología y Cirugía Cardiovascular. Published by Elsevier España, S.L.U. This is an open access article under the CC BY-NC-ND license (http:// creativecommons.org/licenses/by-nc-nd/4.0/).
\end{abstract}

\section{Introducción}

Además de su impacto en el perfil de morbilidad y mortalidad en la población mundial, las arritmias cardiacas causan en los pacientes alteraciones importantes en la calidad de vida relacionada con la salud, generadas principalmente por las limitaciones y los temores que surgen a consecuencia de los síntomas, afectando con ello diferentes dimensiones del ser humano que abarcan desde las áreas física y psicológica, hasta su rol social ${ }^{1}$.

Es así como se ha identificado que este grupo de pacientes experimentan limitaciones en la realización de actividades cotidianas como conducir y dormir ${ }^{2}$, además de marcadas alteraciones en el estado de ánimo dadas por depresión ${ }^{3}$, temor constante y ansiedad anticipatoria relacionada con el hecho de que los síntomas pueden presentarse en cualquier momento ${ }^{4}$. A su vez, se ha detectado que estos pacientes desarrollan comportamientos que interfieren con el ejercicio, las actividades recreativas, la vida social y sus relaciones con personas significativas, además de generar un efecto importante en la vida laboral con repercusiones económicas considerables ${ }^{2-5}$.

Partiendo de las anteriores consideraciones con las que se evidencia el deterioro marcado en la calidad de vida de quienes padecen alteraciones del ritmo cardiaco, se hace imperativo abordar este fenómeno; el primer paso para ello es medir de manera objetiva mediante un instrumento validado, el impacto de los síntomas en la calidad de vida relacionada con la salud; sin embargo, es notorio el vacío relacionado con instrumentos validados en español para tal fin. La escala ASTA ${ }^{5}$ disponible en inglés, permite medir la carga de síntomas generada por las arritmias cardiacas y su impacto en la calidad de vida relacionada con la salud. Dicha escala cuenta con propiedades psicométricas sólidas y puede ser aplicada a personas con diferentes tipos de arritmias, razones que impulsaron, en primera instancia, realizar su adaptación transcultural y con ello obtener la versión en español para posteriormente evaluar sus propiedades de validez y confiabilidad.

El cuestionario $\mathrm{ASTA}^{5}$ en su versión original está conformado por 41 ítems organizados en tres partes o secciones: en la parte I se describen los datos demográficos; la II está conformada por la subescala carga de síntomas específicos de arritmias cardiacas y la parte III por la subescala CVRS que describe la influencia de las arritmias en la situación de vida diaria.

En primer lugar, como resultado de la primera etapa correspondiente al proceso de adaptación transcultural se obtuvo la versión en español del instrumento ASTA, el cual conserva las características conceptuales y semánticas del cuestionario original. Durante este proceso, se siguieron las recomendaciones metodológicas cumpliendo cada una de las fases propuestas en la literatura ${ }^{6-9}$, lo que garantiza el cumplimiento de un proceso que respalda la adaptación adecuada de un idioma a otro y de una cultura a otra.

Una vez culminado el proceso de adaptación transcultural se requirió evaluar las propiedades psicométricas de validez y confiablidad del instrumento ASTA en su versión en español, las cuales son imprescindibles para poder usar los tests de forma rigurosa y científica ${ }^{10}$.

\section{Materiales y método}

\section{Validez}

Esta se evaluó desde los criterios de validez facial o de apariencia, de contenido y de constructo. 
1. Pruebas de validez facial o de apariencia: se refieren a la aceptabilidad que puede tener la escala en el escenario de aplicación ${ }^{11,12}$. La validez facial de la versión en español del instrumento ASTA se determinó en dos etapas: la primera determinada por la evaluación realizada por un grupo de 7 expertos y la segunda por un grupo de personas.

En la primera etapa participaron cuatro docentes del programa de Maestría en Enfermería con énfasis en Salud Cardiovascular de la Universidad Nacional de Colombia y tres enfermeros especialistas con amplia experiencia en el cuidado de pacientes con arritmias cardiacas. Se solicitó al grupo de expertos que evaluaran tres variables en una escala de 1 a 3 para cada uno de los ítems: comprensión, claridad y precisión ${ }^{12}$. El análisis se realizó mediante el modelo de Lawshe modificado, por medio del cual se mide el consenso entre los expertos ${ }^{13}$. El mínimo aceptable para la CVR' es: CVR'=0,5823. Se evaluó mediante este cálculo la validez facial global del instrumento y para cada ítem. Adicionalmente, para esta fase se evaluó la concordancia interobservador por medio del índice de kappa de Fleiss ${ }^{14}$.

La segunda etapa de las pruebas de validez facial correspondió al grupo de personas conformado por 385 hombres y mujeres mayores de 18 años con diferentes niveles de educación y estratos socioeconómicos. Los participantes evaluaron cada uno de los ítems y determinaron para cada uno su comprensibilidad siguiendo los criterios: se comprende, no se comprende muy bien o no se comprende. Para determinar el tamaño de esta muestra se implementó el cálculo del tamaño de la muestra para estimar una proporción ${ }^{15}$. Se determinó el valor de confianza de $95 \%$; la proporción poblacional que comprende el ítem es desconocida por lo cual se asignó el valor de 0,5 y el error máximo admitido es de $5 \%(0,05)$.

2. Pruebas de validez de contenido: buscan que cada uno de los dominios que conforman el área que se está midiendo, esté representado por algún ítem ${ }^{16}$. Para efectuar esta validez nuevamente participaron los expertos convocados para la etapa de validez facial. Para este proceso se evaluaron dos variables siguiendo la recomendación de Polit ${ }^{17}$ : pertinencia y relevancia; la escala de valoración se realizó de 1 a 3 .

Al igual que para la fase de validez facial, en esta fase el análisis de los datos se avaló a partir del acuerdo entre expertos siguiendo del modelo de Lawshe modificado y la concordancia interobservador basada en el modelo de kappa de Fleiss.

3. Prueba preliminar para ajuste: esta fase se desarrolló una vez culminados los procesos de validez facial y de contenido; mediante la prueba preliminar se analizaron dos aspectos fundamentales: particularidades de los ítems y utilidad de la escala ${ }^{6}$.

Para la evaluación relacionada con las particularidades de los ítems se solicitó a los pacientes realizar la evaluación de cada uno de los ítems en los siguientes aspectos: grado de comprensión, ambigüedad, carga afectiva, frecuencia de respuesta y restricción del rango de respuesta. Por otra parte, la utilidad de la escala se evaluó mediante los aspectos de tiempo de diligenciamiento y aplicación requerido, necesidad de entrenamiento y facilidad para calificar el instrumento ${ }^{6}$.

Se aplicó el instrumento a un grupo de 12 pacientes captados en un hospital de cuarto nivel de la ciudad de Bogotá que cumplían con los criterios de inclusión: 1) ser mayor de edad, 2) tener diagnóstico confirmado de arritmia cardiaca, 3 ) tener estado de conciencia y orientación conservados, 4) no presentar alteraciones mentales, 5) no tener dificultad o limitación para la comunicación verbal, 6) estar hemodinámicamente estable y sin complicaciones del estado de salud. Se solicitó autorización al grupo de pacientes para su participación en la prueba preliminar del estudio por medio del consentimiento informado.

4. Validez de constructo: se refiere al grado en que cada prueba refleja el constructo que dice medir, elaborándose operativamente cuando el usuario desea hacer inferencias acerca de conductas o atributos que pueden agruparse bajo la etiqueta de un constructo particular ${ }^{16}$.

Una vez el instrumento contó con validez facial y de contenido, y luego de haber realizado los ajustes necesarios derivados de la prueba preliminar se evaluó la validez de constructo mediante dos técnicas estadísticas: análisis factorial con extracción de componentes principales y análisis de varianza explicada.

Streiner y Norman ${ }^{18}$ sugieren que para efectos del análisis factorial se debe tener un mínimo de 5 individuos por ítem o al menos 100 individuos. La versión en español del instrumento ASTA se aplicó a 202 sujetos que cumplían los criterios de inclusión descritos para la prueba preliminar y quienes presentaban diferentes tipos de arritmias categorizadas en dos grupos: supraventriculares, comprendidas como una serie de ritmos rápidos que tienen su origen superior en la bifurcación del haz de His, y ventriculares definidas como tres o más impulsos ectópicos que se originan por debajo de la bifurcación del haz de His a una frecuencia mayor de 120 latidos por minuto ${ }^{19}$.

Los sujetos fueron captados en los servicios de hemodinámia, urgencias, hospitalización y cardiología de dos hospitales de cuarto nivel de complejidad de la ciudad de Bogotá y se les solicitó autorización para su participación en el estudio por medio de consentimiento informado.

El análisis de datos se desarrolló de manera independiente para cada una de las subescalas del instrumento siguiendo el modelo empleado en la evaluación de validez de constructo del instrumento original.

\section{Confiabilidad}

Las pruebas de confiabilidad se orientaron a evaluar la consistencia interna del instrumento para lo cual se utilizó el coeficiente de alfa de $\mathrm{Cronbach}^{20}$.

\section{Resultados}

\section{Pruebas de validez}

1. Validez facial: en la primera etapa de esta fase, que corresponde a la evaluación por el grupo de expertos, el análisis de cada ítem siguiendo el modelo de Lawshe modificado arrojó que 32 ítems obtuvieron puntaje satisfactorio con valores en un rango entre 0,714 y $1 ; 9$ ítems obtuvieron puntaje menor a 0,5823 , es decir, resultado insatisfactorio, por lo cual se realizaron modificaciones en su redacción sin cambiar el sentido original del ítem siguiendo de manera estricta las recomendaciones del grupo de expertos. 
De manera global, el análisis siguiendo el modelo de Lawshe modificado arrojó un estudio satisfactorio alcanzando 0,777 para la variable de claridad, 0,777 para la de precisión y 0,783 para la de comprensión.

En esta fase, para este mismo grupo de expertos, se evaluó la concordancia interobservador mediante el índice de kappa de Fleiss, en el cual se establece como acuerdo moderado resultados en los rangos $0,41-0,60$, acuerdo sustancial en los rangos 0,61-0,80 y acuerdo casi perfecto 0,81-1,00. De manera global para la variable claridad se obtuvo una concordancia de 0,58 ; para la de precisión de 0,57 y para la de comprensión de 0,58.

Partiendo de las observaciones realizadas por el grupo de expertos en la fase de validez facial, se hicieron modificaciones a la primera versión en español del ASTA a partir de las cuales se generó la segunda versión del instrumento en español.

La segunda etapa de la fase de validez facial, que corresponde al grupo de personas, se aplicó a 395 hombres y mujeres mayores de 18 años con diferentes niveles de educación y estratos socioeconómicos; se obtuvo una comprensibilidad mayor de $95 \%$ para 26 de los 41 ítems, entre el 90 y $94 \%$ para 13 ítems y menor del $90 \%$ para un ítem. A partir de estos resultados, se efectuaron modificaciones a los 14 ítems que obtuvieron comprensibilidad menor de 95\%, ajustando su redacción con la orientación de un literato bilingüe, de manera que fueran más comprensibles sin que perdieran su sentido original. Se obtuvo con ello la tercera versión del instrumento ASTA en español.

Los 14 ítems ajustados fueron evaluados nuevamente mediante su aplicación a 51 personas. Para determinar el tamaño de esta muestra se empleó el cálculo del tamaño de la muestra para estimar una proporción ${ }^{15}$. Se determinó el valor de confianza de 95\%; la proporción poblacional que comprende el ítem es desconocida por lo cual se asignó el valor de 0,888607595 y el error máximo admitido fue de $8,5 \%$ $(0,085)$.

Se recolectó una muestra de 58 personas, hombres y mujeres, mayores de 18 años, de diferentes niveles de escolaridad y estratos socioeconómicos. Resultado de esta evaluación, se obtuvo una comprensibilidad mayor del 95\% para 6 de los 14 ítems ajustados; entre el 90 y 94\% para 5 y menor del $90 \%$ para 3 de los ítems por lo cual se decidió eliminarlos del instrumento.

Como resultado de la evaluación de validez facial de los ítems ajustados, surgió la cuarta versión en español del instrumento ASTA que consta de 37 ítems de los cuales 31 cuentan con comprensibilidad mayor o igual al $95 \%$ y 6 entre 90 y $94 \%$.

2. Validez de contenido: El análisis bajo el modelo modificado propuesto por Lawshe ${ }^{13}$ arrojó los siguientes resultados: para el criterio de pertinencia, 35 de los 41 ítems alcanzaron un nivel de concordancia absoluta con un puntaje de $1 ; 5$ ítems lograron un nivel satisfactorio con un puntaje de 0,857 y uno obtuvo un nivel de concordancia menor de 0,58 . En lo concerniente al criterio de relevancia, se obtuvieron 33 ítems con $100 \%$ de concordancia, es decir, con puntuación de $1 ; 7$ alcanzaron puntuación de 0,857 , considerada satisfactoria y 1 obtuvo puntaje de 0,58 , considerada no satisfactoria.
El análisis de concordancia interobservador se avaló a partir del índice de kappa de Fleiss ${ }^{14}$ obteniendo los siguientes resultados de manera global: para la variable de relevancia, se obtuvo un resultado de 0,91 , en tanto que para la variable pertinencia un resultado de 0,93 , lo que corresponde a un acuerdo casi perfecto entre los expertos.

El ítem 13 obtuvo un valor de 0,0428 para los criterios de relevancia y pertinencia lo que corresponde a un acuerdo moderado entre los expertos, razón por la cual se decidió eliminar del instrumento.

La versión final del instrumento ASTA resultado de los procesos de validez facial y de contenido consta de 36 ítems y fue evaluado por un literato bilingüe, quien verificó la adecuación contextual y semántica para ser aplicada en Colombia. Resultado de esta revisión se determinó que esta versión en español cuenta con una adecuada comprensibilidad para el colombiano promedio.

3. Prueba preliminar para ajustes: participaron 12 pacientes que cumplen con los criterios de inclusión determinados, quienes aceptaron participar en esta fase del estudio mediante consentimiento informado y no hicieron parte de la muestra determinada para realizar la validez de constructo. Este grupo de pacientes se captó en un hospital de cuarto nivel de la ciudad de Bogotá en los servicios de hospitalización, urgencias, cardiología, consulta externa y electrofisiología.

Luego de realizar la prueba preliminar se estimó que el tiempo necesario para diligenciar el instrumento estuvo entre 10 y 15 minutos; la totalidad de los participantes afirmaron que todos los ítems se comprenden claramente, los ítems están redactados en forma precisa de tal manera que no se prestan para diversas interpretaciones, también afirmaron que ni las preguntas ni las opciones de respuesta contienen adjetivos que induzcan una respuesta determinada.

Se indagó a cada uno de los participantes de la prueba preliminar si consideraban que algún ítem era igual a otro, a lo cual todos respondieron "no". En cuanto a la utilidad de la escala, se evidenció que puede ser aplicada por profesionales con un entrenamiento básico orientado a explicar el objetivo de la medición.

4. Validez de constructo: se captó por una muestra de 202 pacientes que cumplieron con los criterios de inclusión establecidos y que correspondió a una relación de 8,4 participantes por ítem (tabla 1).

Durante esta fase se encontró el hecho de que al aplicar el instrumento a los participantes, indicaron no encontrar diferencia en dos de las opciones de respuesta ( $\mathrm{Si}$, mucho y $\mathrm{Si}$, bastante) razón por la que se decidió eliminar una opción de repuesta y con ello reducir el riesgo de una medición equivocada, pues es conocido que si se ofrecen alternativas de respuesta en las que el sujeto tenga problemas para discriminar entre ellas, hay mayor probabilidad de introducción de errores de medición ${ }^{21}$. Esta modificación no afectará de manera negativa la validez de constructo ni la confiabilidad del instrumento.

En las tablas 2 y 3 se exponen los resultados obtenidos relacionados con la varianza explicada y el análisis de factores con extracción de componentes principales utilizando tres opciones de respuesta. 
Tabla 1 Características demográficas, muestra validez de constructo y confiabilidad

\begin{tabular}{|c|c|c|c|c|c|c|c|c|c|c|}
\hline Diagnóstico y pacientes & Total $n=202$ & TSVRN $n=33$ & WPW $n=13$ & $F A n=76$ & FLA $n=10$ & TA $n=3$ & $\mathrm{TV} n=47$ & TS $n=11$ & $\begin{array}{l}\text { Aleteo } \\
\text { ventricular } \\
\mathrm{n}=1\end{array}$ & $\begin{array}{l}\text { Extrasístoles } \\
\text { ventricula- } \\
\text { res } n= \\
8\end{array}$ \\
\hline Edad promedio (desviación estándar) & $55.1(18.1)$ & $55.7(17.7)$ & $33.1(11.1)$ & 66,2 (11.7) & $45.4(13.4)$ & $79.6(5.6)$ & $56.6(13.9)$ & $33.6(21.5)$ & 53 & $42.7(19,9)$ \\
\hline $\begin{array}{l}\text { Género F/M } \\
\text { (\%) }\end{array}$ & $\begin{array}{l}113 / 90 \\
(56 / 44)\end{array}$ & $\begin{array}{l}28 / 5 \\
(82 / 18)\end{array}$ & $\begin{array}{l}5 / 8 \\
(38 / 62)\end{array}$ & $\begin{array}{l}40 / 36 \\
(53 / 47)\end{array}$ & $\begin{array}{l}9 / 1 \\
(90 / 10)\end{array}$ & $\begin{array}{l}3 / 0 \\
(100 / 0)\end{array}$ & $\begin{array}{l}14 / 33 \\
(30 / 70)\end{array}$ & $\begin{array}{l}8 / 3 \\
(73 / 27)\end{array}$ & $\begin{array}{l}1 / 0 \\
(100 / 0)\end{array}$ & $\begin{array}{l}4 / 4 . \\
(50 / 50)\end{array}$ \\
\hline $\begin{array}{l}\text { Convive/ vive sólo } \\
\text { (\%) }\end{array}$ & $\begin{array}{l}194 / 9 \\
(96 / 4)\end{array}$ & $\begin{array}{l}32 / 1 \\
(97 / 3)\end{array}$ & $\begin{array}{l}13 / 0 \\
(100 / 0)\end{array}$ & $\begin{array}{l}72 / 4 \\
(95 / 5)\end{array}$ & $\begin{array}{l}10 / 0 \\
(100 / 0)\end{array}$ & $\begin{array}{l}3 / 0 \\
(100 / 0)\end{array}$ & $\begin{array}{l}45 / 2 \\
(96 / 4)\end{array}$ & $\begin{array}{l}9 / 2 \\
(82 / 18)\end{array}$ & $\begin{array}{l}1 / 0 \\
(100 / 0)\end{array}$ & $\begin{array}{l}8 / 0 \\
(100 / 0)\end{array}$ \\
\hline \multicolumn{11}{|l|}{ Educación } \\
\hline Primaria (\%) & $86(42)$ & $11(32)$ & $4(31)$ & $36(47)$ & $3(30)$ & $1(33)$ & 23(49) & $4(36)$ & $0(0)$ & $3(36)$ \\
\hline Secundaria (\%) & $63(31)$ & $5(18)$ & $5(38)$ & $27(36)$ & $2(20)$ & $1(33)$ & $16(34)$ & $5(46)$ & $1(100)$ & $0(0)$ \\
\hline Universitario (\%) & $54(27)$ & $17(50)$ & $4(31)$ & $13(17)$ & $5(50)$ & $1(33)$ & $8(17)$ & $21(18)$ & $0(0)$ & $5(64)$ \\
\hline \multicolumn{11}{|l|}{ Ocupación } \\
\hline Empleado (\%) & $62(30)$ & $12(35)$ & $8(62)$ & $17(22)$ & $4(40)$ & $0(0)$ & $11(23)$ & $2(18)$ & $0(0)$ & $4(50)$ \\
\hline Independiente (\%) & $32(16)$ & $4(12)$ & $2(15)$ & $8(11)$ & $1(10$ & $1(33)$ & $14(30)$ & $0(0)$ & $0(0)$ & $2(25)$ \\
\hline Hogar (\%) & $38(19)$ & $8(23)$ & $1(8)$ & $17(22)$ & $4(40)$ & $1(33)$ & $4(9)$ & $2(18)$ & $1(100)$ & $0(0)$ \\
\hline Cesante (\%) & $58(29)$ & $2(9)$ & $0(0)$ & $34(45)$ & $1(10)$ & $1(33)$ & $17(36)$ & $2(18)$ & $0(0)$ & $0(0)$ \\
\hline Estudiante (\%) & $13(6)$ & $7(21)$ & $2(15)$ & $0(0)$ & $0(0)$ & $0(0)$ & $1(2)$ & $5(46)$ & $0(0)$ & $2(25)$ \\
\hline
\end{tabular}

FA=Fibrilación auricular; FLA= flutter auricular; $T A=$ taquicardia auricular; $T S=$ taquicardia sinusal $\mathrm{TSVRN}=$ taquicardia supraventricular por reentrada nodal; $\mathrm{TV}=$ taquicardia ventricular; WPW= síndrome de Wolff-Parkinson-White.

Fuente: Datos de la investigación. 
Tabla 2 Varianza total explicada escala carga de síntomas

\begin{tabular}{|c|c|c|c|c|c|}
\hline \multirow[t]{2}{*}{ Componente } & \multicolumn{3}{|c|}{ Autovalores iniciales } & \multicolumn{2}{|c|}{$\begin{array}{l}\text { Sumas de extracción } \\
\text { de cargas al cuadrado }\end{array}$} \\
\hline & Total & $\%$ de varianza & $\%$ acumulado & Total & $\%$ de varianza \\
\hline 1 & 4,791 & 43,558 & 43,558 & 4,791 & 43,558 \\
\hline 2 & 1,230 & 11,182 & 54,740 & & \\
\hline 3 & 1,109 & 10,080 & 64,820 & & \\
\hline 4 & 967 & 8,791 & 73,611 & & \\
\hline 5 & ,812 & 7,377 & 80,989 & & \\
\hline 6 & ,613 & 5,573 & 86,561 & & \\
\hline 7 &, 504 & 4,578 & 91,139 & & \\
\hline 8 & ,423 & 3,841 & 94,980 & & \\
\hline 9 & , 301 & 2,739 & 97,719 & & \\
\hline 10 & ,161 & 1,466 & 99,186 & & \\
\hline 11 & ,090 &, 814 & 100,000 & & \\
\hline
\end{tabular}

Fuente: Datos de la investigación.

Tabla 3 Varianza total explicada escala calidad de vida relacionada con la salud

\begin{tabular}{|c|c|c|c|c|c|}
\hline \multirow[t]{2}{*}{ Componente } & \multicolumn{3}{|c|}{ Autovalores iniciales } & \multicolumn{2}{|c|}{$\begin{array}{l}\text { Sumas de extracción } \\
\text { de cargas al cuadrado }\end{array}$} \\
\hline & Total & $\%$ de varianza & $\%$ acumulado & Total & $\%$ de varianza \\
\hline 1 & 5,280 & 40,617 & 40,617 & 5,280 & 40,617 \\
\hline 2 & 1,182 & 9,090 & 49,707 & & \\
\hline 3 & 948 & 7,296 & 57,003 & & \\
\hline 4 & 873 & 6,712 & 63,715 & & \\
\hline 5 & 720 & 5,541 & 69,256 & & \\
\hline 6 & 696 & 5,356 & 74,612 & & \\
\hline 7 & 661 & 5,084 & 79,696 & & \\
\hline 8 & 546 & 4,200 & 83,896 & & \\
\hline 9 & 512 & 3,939 & 87,835 & & \\
\hline 10 & 474 & 3,643 & 91,478 & & \\
\hline 11 & 432 & 3,323 & 94,801 & & \\
\hline 12 & 370 & 2,844 & 97,645 & & \\
\hline 13 & 306 & 2,355 & 100,000 & & \\
\hline
\end{tabular}

Fuente: Datos de la investigación.

Resultado de este análisis se observa que para la subescala carga de síntomas el $43,5 \%$ de la varianza total está contenida en un factor; lo mismo puede afirmarse para la subescala CVRS en la que el 40,6\% de la varianza total está contenida en un factor.

El análisis factorial fue hecho con el paquete SPSS versión 19; se utilizó el método de extracción por componentes principales, bajo el criterio de Kaiser y la rotación varimax, para las dos escalas: carga de síntomas y CVRS; el análisis se hizo de manera independiente para cada una de ellas siguiendo el modelo del estudio original y se obtuvieron los resultados expuestos en las tablas 4 y 5 .

\section{Confiabilidad}

Para analizar la consistencia interna como un elemento de la confiabilidad, se usaron dos coeficientes para cada una de las escalas: alpha de Cronbach y coeficiente de correlación en la prueba de las dos mitades coeficiente de Guttman
Tabla 4 Matriz de componentes (cargas factoriales) escala carga de síntomas

\begin{tabular}{ll}
\hline & Componente \\
& 1 \\
\hline Ítem 6a &, 749 \\
Ítem 6b &, 746 \\
Ítem 6c &, 657 \\
Ítem 6d &, 699 \\
Ítem 6e &, 714 \\
Ítem 6f &, 746 \\
Ítem 6g &, 667 \\
Ítem 6h &, 701 \\
Ítem 6i &, 478 \\
Ítem 7 &, 593 \\
Ítem 8 &, 413 \\
\hline
\end{tabular}

Fuente: Datos de la investigación. 
Tabla 5 Matriz de componentes (cargas factoriales) escala calidad de vida relacionada con la salud

\begin{tabular}{ll}
\hline & Componente \\
& 1 \\
\hline Ítem 1 &, 709 \\
Ítem 2 &, 690 \\
Ítem 3 &, 621 \\
Ítem 4 &, 647 \\
Ítem 5 &, 649 \\
Ítem 6 &, 668 \\
Ítem 7 &, 736 \\
Ítem 8 &, 630 \\
Ítem 9 &, 599 \\
Ítem 10 &, 545 \\
Ítem 11 &, 444 \\
Ítem 12 &, 769 \\
Ítem 13 &, 497 \\
\hline
\end{tabular}

Fuente: Datos de la investigación.

(asociado a esta prueba se encuentra el coeficiente de Spearman-Brown). Las tablas 6 y 7 presentan los valores de los coeficientes anteriormente nombrados.

\section{Discusión}

Se logra una versión en español del instrumento ASTA el cual cuenta con propiedades psicométricas satisfactorias que evidencian su validez y confiabilidad. Este instrumento basado en el modelo de Calidad de Vida Relacionado con la Salud de Wilson y Clearly ${ }^{22}$ mide la carga de síntomas y su impacto en la calidad de vida relacionado con la salud en pacientes con arritmias cardiacas, de tal manera que las puntuaciones más altas obtenidas con esta escala indican mayor carga de síntomas y mayor impacto negativo en la calidad de vida relacionada con la salud.

\section{Utilidad desde la medición}

La versión del instrumento ASTA en español cuenta con validez facial, evidenciada por un nivel de concordancia satisfactorio. Los ítems que obtuvieron un valor no satisfactorio requirieron de un ajuste en su redacción y de esta manera se adaptaron a nuestra cultura. Uno de los ítems fue eliminado por considerarlo inconsistente.

Mediante la evaluación de kappa de Fleiss se obtuvo para las tres variables un acuerdo moderado, hecho que puede explicarse por la redacción de los ítems, pues en ellos los expertos sugirieron diversas aclaraciones y modificaciones.

En general, como resultado de las pruebas de validez facial con el grupo de expertos se evidencia que los ítems que constituyen el instrumento ASTA en su versión en español cuentan con un adecuado entendimiento, se emplea un lenguaje apropiado en su redacción y están expresados de manera concisa y exacta, evitando ambigüedades.

En relación con la evaluación realizada en el grupo de personas para esta misma fase de validez, se observó la necesidad de ajustar 14 ítems por no obtener comprensibilidad adecuada, lo que puede deberse al uso de palabras muy técnicas ajenas al vocabulario del colombiano promedio. Al final de este proceso, los resultados arrojaron una comprensibilidad adecuada, obteniendo un valor mayor a $95 \%$ para 31 ítems y de $90 \%$ a $94 \%$ para 6 ítems.

Tabla 6 Coeficientes consistencia interna escala de carga de síntomas

\begin{tabular}{llll}
\hline Alpha de Cronbach & Parte 1 & Valor & 0,848 \\
& & N de elementos & 6 \\
& Parte 2 & Valor & 0,682 \\
& & N de elementos & 5 \\
& N total de elementos & & 11 \\
Coeficiente de & Correlación entre formularios & & 0,625 \\
Spearman-Brown & Longitud igual & 0,769 \\
Coeficiente de dos mitades de Guttman & Longitud desigual & 0,770 \\
\hline
\end{tabular}

Fuente: Datos de la investigación.

Tabla 7 Coeficientes consistencia interna subescala de calidad de vida relacionada con la salud

\begin{tabular}{llll}
\hline Alpha de Cronbach & Parte 1 & Valor & 0,832 \\
& & N de elementos & 7 \\
& Parte 2 & Valor & 0,722 \\
& & N de elementos & 6 \\
& N total de elementos & & 13 \\
Coeficiente de & Correlación entre formularios & 0,727 \\
Spearman-Brown & Longitud igual & 0,842 \\
Coeficiente de dos mitades de Guttman & Longitud desigual & & 0,843 \\
\hline
\end{tabular}

Fuente: Datos de la investigación. 
De otra parte, se confirmó la validez de contenido de la versión del instrumento ASTA en español validado en una concordancia satisfactoria de los expertos evaluada por medio de los modelos de Lawshe modificado y kappa de Fleiss. En el primero de ellos, de un total de 41 ítems se obtuvo 40 con puntaje satisfactorio o perfecto en cada uno de los criterios (pertinencia y relevancia), en tanto que la evaluación bajo el segundo modelo, arrojó un acuerdo casi perfecto para estos mismos criterios. Los resultados obtenidos en la fase de evaluación de validez de contenido indican que los ítems del instrumento son pertinentes y relevantes para medir los constructos carga de síntomas y calidad de vida relacionada con la salud.

De otro lado, la validez de constructo del instrumento ASTA evidencia un solo factor para cada una de las subescalas del instrumento. De estos resultados se puede afirmar que el análisis factorial reveló la presencia de un solo factor para cada una de las subescalas, lo cual significa que relaciones entre cada uno de los ítems pueden explicarse a partir de una sola variable no observable (latente), es decir, de un solo factor. Significa, así mismo, que todos los ítems de la subescala carga de síntomas son pertinentes para medir únicamente la carga de los síntomas. De igual forma, todos los ítems de la subescala calidad de vida relacionada con la salud son pertinentes para medir únicamente el ítem calidad de vida relacionada con la salud.

De otra parte, en relación con el análisis de varianza para la evaluación de validez de constructo, $\mathrm{Cea}^{23}$ recomienda un valor de $30 \%$. En el caso de las dos subescalas con solo el primer componente se cumple el porcentaje de varianza de $30 \%$, lo que significa que para la escala carga de síntomas el $43,5 \%$ de la varianza en la puntuación obtenida está explicado desde este único factor. De igual manera, para el caso de la escala calidad de vida relacionada con la salud el $40,6 \%$ de la varianza en la puntuación obtenida está explicado desde este único factor. Esta verificación estadística se considera como valor adecuado.

Por otro lado, se demuestra una consistencia interna muy satisfactoria a partir de los resultados obtenidos, los cuales muestran alto grado de homogeneidad para cada una de las escalas, es decir, que las respuestas son lo suficientemente coherentes (relacionadas entre sí) como para poder concluir que todos los ítems miden lo mismo, y por tanto son sumables en una puntuación total única que representa, mide un rasgo.

Una dificultad que surgió durante la aplicación de la versión en español del instrumento se relaciona con las opciones de respuesta, pues para muchos pacientes no hay diferencia entre las opciones de respuesta "Si, mucho" y "Si, bastante", por tanto se decidió eliminar una de ellas con lo cual se modificó el puntaje obtenido, situación que fue informada a la autora y frente a la cual se espera en próximas aplicaciones incluir otra opción de respuesta que logre marcar diferencia respecto a las ya existentes.

De otra parte, es importante mencionar que el instrumento ASTA en su versión en español permite indagar el impacto que generan los tratamientos farmacológicos o intervencionistas en la calidad de vida relacionada con la salud.

\section{Utilidad desde la práctica disciplinar}

Esta se obtiene de manera fundamental a partir de la identificación de los factores determinantes de la calidad de vida relacionados con la salud que se ven afectados por los síntomas que generan las arritmias cardiacas, lo que se logra mediante el uso de un instrumento válido y confiable, adaptado a nuestra cultura.

Poner a disposición un instrumento adaptado a la cultura colombiana, válido y confiable, que permita medir la calidad de vida relacionada con la salud en personas con arritmia cardiaca, constituye el punto de partida del abordaje de este fenómeno; con él se pretende obtener información que evidencie las áreas que requieren ser contempladas por los profesionales de enfermería y de otras áreas de la salud, en aras de disminuir los marcados efectos negativos en la calidad de vida relacionada con la salud que ocasionan las síntomas experimentados durante las arritmias cardiacas, procurando un trabajo interdisciplinario con el objetivo de trascender a un campo que ha permanecido relegado y desconocido tal vez por la preocupación predominante de los profesionales de la salud por el conocimiento y el desarrollo de destrezas que demandan el manejo de las tecnologías disponibles para su tratamiento.

\section{Conclusiones}

La versión del instrumento ASTA en español cuenta con propiedades psicométricas que lo hacen válido y confiable para medir el impacto de los síntomas en la calidad de vida relacionada con la salud de los pacientes con arritmias cardiacas.

Esta investigación se configura como el punto de partida para el estudio del impacto de síntomas en la calidad de vida relacionada con la salud de los pacientes con arritmias cardiacas, pues con la información obtenida a partir de su implementación se logran identificar las áreas que requieren intervención (manejo de los síntomas, enfrentamiento a la enfermedad, educación sobre estas alteraciones del ritmo cardiaco y su tratamiento, terapias cognitivas, intervenciones de regulación emocional, fomento y orientación de apoyo familiar, creación de programas de atención integral dentro de los cuales se conformen grupos que se conviertan en espacios de socialización e interacción entre los diferentes participantes). Se pretende, por tanto, diseñar e implementar estrategias que involucren un trabajo interdisciplinario tendiente a manejar los síntomas y a mitigar el efecto negativo que estos generan en la calidad de vida relacionada con la salud.

\section{Conflictos de interés}

Ninguno.

\section{Bibliografía}

1. Walfridsson $U$, Walfridsson $H$. The impact of supraventricular tachycardias on driving ability in patients referred for radiofrequency catheter ablation. PACE. 2005;28:191-5.

2. Wood k, Stewart A, Drew B, Scheinman M, Frolicher E. Development and initial psychometric evaluation of the patient 
perspective of arrhythmia questionnaire. Res Nurs Health. 2009;32:504-16.

3. Suzuki S, Kasanuki K. The influences of psychosocial aspect and anxiety symptoms on quality of life of patients with arrhythmia: investigation in paroxysmal atrial fibrillation. Int. J Behav Med. 2004;11:104-9.

4. McCabe P, Schumacher K, Barnason S. Living with atrial fibrillation: A Qualitative study. J Cardiovasc Nurs. 2011;18: 431-44.

5. Walfridsson U. Assessing Symptom Burden and Health-Related Quality of Life in patients living with arrhythmia and ASTA Arrhythmia-Specific questionnaire in Tachycardia and Arrhythmia [dissertation]. Linköping University, Division of Nursing Science; 2011.

6. Sánchez R, Gómez C. Conceptos básicos sobre validación de escalas. Rev Colomb Psiquiatría. 1998;27:122.

7. Ramada-Rodilla J. Adaptación cultural y validación de cuestionario de salud: revisión y recomendaciones metodológicas. Salud Pública Méx. 2013;55:57-66.

8. Gaite Luis. Traducción y adaptación transcultural de instrumentos de evaluación en psiquiatría: aspectos metodológicos. Arch Neurobiol. 1997;60:91-111.

9. Guillemin F. Cross-cultural adaptation and validation of health status measures. Scand J Rheumatol. 1995;24: 61-3.

10. Muñiz J. Las teorías de los test: Teoría clásica y teoría de respuesta a los ítems. Papeles del Psicólogo. 2010;3: 57-66.

11. Feinstein A. Clinimetrics. Yale University Press; 1987.

12. Herrons L. El desarrollo de las pruebas psicométricas. En: Psicología y medición. México: Limusa; 1985. p. 78.
13. Tristán A. Modificación al modelo de Lawshe para el dictamen de validez de contenido de un instrumento objetivo. Avances en Medición. 2008;6:37-48.

14. Fleiss J. The design and analysis of clinical experiments. New York: Wiley; 1999. p. 44.

15. Fuentelsaz C. Cálculo del tamaño de la muestra. Matronas Prof. 2004;5:5-13.

16. Tovar J. Psicometría: tests psicométricos, confiabilidad y validez. En: Psicología: Tópicos de Actualidad. Lima: Universidad Nacional Mayor de San Marcos. Facultad de Psicología; 2007:85-108.

17. Polit D, Hungler B. Análisis de los datos cuantitativos. En: Investigación científica en ciencias de las salud: Diseños de investigación para estudios cuantitativos. Quinta edición. México: McGraw Hill; 2010. p. 276-343.

18. Streiner L, Norman G. Health measurement scales: a practical guide to their development and use. Oxford: Oxford University Press; 1995.

19. Camacho H, Gutiérrez de Piñeres O, Duque M, Gómez-Ortiz A. Arritmias ventriculares. Bogotá: Guías de práctica clínica basadas en la evidencia. ISS-ASCOFAME. 1997.

20. Cronbach L. Coefficient alpha and the internal structure of test: Psychometrika. 1951;16:297-334.

21. Lozano L, García-Cueto E, Muñiz J. Effect of the number of response categories on the reliability and validity of rating scales. Methodology. 2008;4:73-9.

22. Wilson I, Cleary P. Linking clinical variables with health-related quality of life. A conceptual model of patient outcomes. JAMA. 1995;273:59-65.

23. Cea DA. Análisis multivariable. Teoría y práctica en la investigación social. Madrid: Síntesis; 2002. p. 207-9. 